\title{
Höhere Thrombosegefahr mit neuen oralen Kontrazeptiva
}

Das Risiko, eine venöse Thromboembolie zu entwickeln, ist mit den meisten oralen Kontrazeptiva der dritten Generation höher als mit älteren Kombinationspräparaten.

Alle zur Verhütung eingesetzten oralen Östrogen-Gestagen-Kombinationen begünstigen die Entstehung von Thrombosen. Für Kontrazeptiva der dritten Generation gab es bisher aber kaum Zahlen. Zwei Fall-Kontroll-Studien aus Großbritannien zeigen nun, dass die enthaltenen Gestagene keineswegs sicherer sind als die Vorläufersubstanzen. Mit Gestoden, Drospirenon, Cyproteron und Desogestrel lag das Thromboserisiko sogar um 50-80\% höher als mit Levonorgestrel.

In die Studien einbezogen wurden Frauen im Alter zwischen 15 und 49, die zwischen 2001 und 2013 in einer von zwei britischen Patientendatenbanken - Clinical Practice Research Datalink (CPRD) und QResearch - erfasst worden waren. 5062 bzw. 5500 Patientinnen mit einer venösen Thromboembolie (VTE) wurden 19.638 bzw. 22.396 vergleichbare Frauen ohne ein solches Ereignis gegenübergestellt. Die VTE-Patientinnen waren nicht nur öfter Raucherinnen und adipös, sie hatten im Jahr vor dem Indexdatum auch häufiger orale Kontrazeptiva verschrieben bekommen als die Kontrollen (30\% vs. $18 \%$ in CPRD bzw. $28 \%$ vs. $16 \%$ in QResearch).

\section{VTE-Risiko teilweise vervierfacht}

Die Ergebnisse der beiden Fall-KontrollStudien zusammengenommen, hatten Frauen unter oraler Kontrazeption ein rund dreimal so hohes Risiko für eine VTE wie Frauen, die innerhalb des zurückliegenden Jahres nicht mit der Pille verhütet hatten (adjustierte Odds Ratio, aOR 2,97). Dabei ließen sich die verwendeten Gestagene klar in zwei Gruppen teilen. In Gruppe 1 war das Risiko etwa 2,5-fach erhöht, dazu gehörten Levonorgestrel (aOR 2,38), Norethisteron (OR 2,56) und Norgestimat (aOR 2,53). In Gruppe 2 kam es ungefähr zu einer Vervierfachung des Risikos, die Substanzen waren Desogestrel (aOR 4,28),
Gestoden (aOR 3,64), Drospirenon (aOR 4,21) und Cyproteron (aOR 4,27).

\section{Levonorgestrel und Norgestimat am sichersten}

Die niedrigste Zahl zusätzlicher VTE pro 10.000 Frauen und Jahr ist nach dieser Analyse mit Levonorgestrel und Norgestimat zu erwarten; sie belief sich auf jeweils 6 bei den 15 - bis 49 - und jeweils 7 bei den 25- bis 49-Jährigen. Die höchste Zahl von hormonassoziierten VTE wurde im Alter von 15-49 mit Desogestrel und Cyproteron gesehen, mit jeweils 14 Fällen pro 10.000 Frauen und Jahr. Im Alter von 25-49 kam es in Zusammenhang mit Drospirenon, Desogestrel und Cyproteron sogar zu jeweils 17 Extra-VTE pro 10.000 Frauen und Jahr.

Ein signifikant erhöhtes Thromboserisiko im Zusammenhang mit einer vor Kurzem begonnenen hormonellen Verhütung war nur für Levonorgestrel zu erkennen. Die Dosierung des Östrogens, die nur in Kombination mit drei Gestagenen untersucht werden konnte, zeigte keine Auswirkung auf die VTE-Rate.

(Beateschumacher)

Vinogradova Y et al. BMJ 2015;350:h2135

\section{Vollrausch im Mutterleib: Ruf nach mehr Prävention}

Fast jede fünfte Schwangere trinkt während der Schwangerschaft Alkohol und riskiert damit bei ihrem Kind bleibende Schäden.

Nach Daten des Robert Koch-Instituts in Berlin trinkt fast jede fünfte Schwangere Alkohol. Die Folge: Bundesweit werden jährlich 10.000 Kinder mit Fetalen Alkoholspektrum Störungen (FASD) geboren, davon etwa 2000 schwer geschädigte Kinder. Die Beeinträchtigungen reichen dabei von leichten Konzentrationsproblemen bis zu starken Schäden in der geistigen und motorischen Entwicklung sowie Wachstumsstörungen und Gesichtsfehlbildungen. Ihr Schicksal wäre vermeidbar, meinen Experten. Die Behinderungen wären vermeidbar, wenn Schwangere abstinent blieben. Politiker aus CDU und SPD haben daher kürzlich für Warnhinweise auf Alkoholika plädiert. Generell sind bei FASD mehr Prävention, Aufklärung, Diagnose und Behandlung nötig. Nach einer aktuellen Umfrage wissen nur $44 \%$ der Deutschen über die bleibenden Schäden durch Alkohol in der Schwangerschaft Bescheid.

Ziel ist es besonders auch, betroffene Kinder früh zu erkennen, um Hilfe, Förderung und Kontrolle zu etablieren. Tatsächlich wird laut den Sachverständigen nur etwa ein Fünftel der Fälle sofort erkannt. Mit Gesetz und Strafe ist dem Thema nicht beizukommen: Selbstschädigung ist in Deutschland nicht verboten, und eine Fremdschädigung liegt nicht vor, weil Kinder juristisch erst ab der Geburt ein eigenes Leben haben.

(eis)

dpa; www.aerztezeitung.de 